\title{
Matrix Training and Verbal Generativity in Children with Autism
}

\author{
Kelly T. Kohler • Richard W. Malott
}

Published online: 18 July 2014

(C) Association for Behavior Analysis International 2014

\section{Three-Dimensional Matrix Training and Verbal Generativity in Children with Autism}

As verbal beings, we can produce and respond to statements never said or heard before. This is perhaps the most unique and important feature of language and has been referred to as generative language (e.g., Goldstein 1984; Lutzker and Sherman 1974; Stewart, McElwee, and Ming 2013), linguistic productivity (Hockett 1960; Malott 2003; Whaley and Malott 1971), generative grammar (Chomsky 1959), or recombinative generalization (e.g., Goldstein 1983a; Goldstein 1983b; Goldstein 1984; Goldstein and Brown 1989; Goldstein and Mousetis 1989). According to Lutzker and Sherman (1974), "generative language simply means the appearance of novel language responses within the language repertoire of the child that have not been modeled or directly trained, but that may be related to other language responses" (p. 447). The basic behavioral processes underlying generative language have yet to be clarified to everyone's satisfaction, but generative language should not be confused with simple stimulus or

This article is based on Kelly T. Kohler's Master's project. We wish to give special thanks to her committee members, Stephanie Peterson and Ron Van Houten, for their helpful support.

K. T. Kohler $\cdot$ R. W. Malott $(\bowtie)$

Department of Psychology, Western Michigan University,

Kalamazoo, MI 49008, USA

e-mail: dickmalott@dickmalott.com

K. T. Kohler

e-mail: kellytstone@gmail.com response generalization because correct novel response sequences are no more physically similar to the training sequences than are incorrect response sequences (Stewart et al. 2013).

Acquisition of sentence structures (or autoclitic frames; Skinner 1957, p. 336) can enable the construction of novel sentences composed of tacts already in the repertoire without direct training (Mackay and Fields 2009). Matrix training is a teaching tool that might be used to teach such sentence structures and thereby facilitate generative responding (e.g., Axe and Sainato 2010; Goldstein 1983a; Goldstein 1983b; Goldstein 1984; Goldstein and Brown 1989; Goldstein and Mousetis 1989; Yamamoto and Miya 1999).

With matrix training, individual components of a sentence are arranged along each axis of a matrix and are combined to form phrases or sentences. As an example, verbs such as kick, throw, and drop can be listed along one axis and objects such as ball, block, and book along another axis to form nine phrases: kick ball, throw ball, drop ball, kick block, and so on. Rather than teaching all nine phrases, only the phrases along a diagonal of the matrix are taught (e.g., kick ball, throw block, and drop book). Thus, each individual word is trained without repetition, but the words are taught in a number of combinations so that the remaining phrases might occur without direct training. In addition, it is possible that other phrases involving known words that have not been involved in the matrix training might occur.

Previous research on matrix training has involved non-sense words referring to color-shape combinations 
(Esper 1925), expressive and receptive object-location and object-preposition-location sentences (Goldstein and Brown 1989; Goldstein and Mousetis 1989), subject-verb-object sentence construction (Yamamoto and Miya 1999), sociodramatic play (Dauphin, Kinney, and Stromer 2004), and receptive actionobject directions (Axe and Sainato 2010). Many previous studies have involved nonsense word combinations (e.g., Esper 1925; Foss 1968; Goldstein 1983a; Goldstein 1983b; Goldstein 1984).

The current study involves teaching tacts in the form of subject-verb-object ( $\mathrm{S}-\mathrm{V}-\mathrm{O}$ ) sentences. In keeping with the practitioner model (Malott et al. 2011), the primary goal was that the children benefit from their participation. Therefore, we used words for common objects found in their everyday environments. The children could already emit these words as single-word tacts, but could not emit S-V-O sentence tacts in which the single-word tacts were combined. We used 162 short videos combining subjects, verbs, and objects (e.g., "Hunter kicks ball" and "Jake throws block") for training and testing. The research question was: What are the effects of matrix training on generative S-V-O tacts?

\section{Method}

Participants and Setting

The participants were two 5-year-old children diagnosed with ASD who attended an early intervention center for children with autism and other disabilities. At the start of this study, they could emit 1 to 3 word mands and tacts, including some subject-verb combinations (see Table 1 for a description of their mand and tact repertoires). They could also answer some basic questions (e.g., "What is your name?" "How old are you?" "What are some foods?"). However, other than "I want __," neither of the children had emitted generative sentences.

\section{Materials}

We used six three-dimensional S-V-O matrices (see Fig. 1) and 27 possible sentences in each for a total of 162 sentences. All $162 \mathrm{~S}-\mathrm{V}-\mathrm{O}$ videos were created using familiar people and objects. They were saved into PowerPoint files in random order to be used in all phases of the study.
Experimental Design

We used a multiple probe design across responses to assess generative transfer within and across matrices. After training on a subset of responses within a particular matrix, we probed the remaining responses within that matrix. If generative transfer within the matrix occurred, we conducted probes with the remaining matrices.

\section{Interobserver Agreement}

Tutors who worked with the children collected interobserver agreement data during the sessions or later via videos. For Shreeya, interobserver agreement was assessed for $68.8 \%$ of sessions, with a mean agreement of $99.8 \%$, and a session range of 96 to $100 \%$. For Jake, interobserver agreement was assessed for $81.1 \%$ of sessions, with a mean agreement of $99.8 \%$, and a session range of 83 to $100 \%$.

Procedure

Pre-training We tested 18 subjects, 18 verbs, and 18 objects across six matrices to ensure that the individual words in the sentences were in the children's repertoires. We presented pictures of the subjects and objects and videos of the actions and said "What" or "What is it?" If a child did not correctly tact a noun or verb within $3 \mathrm{~s}$ of the first presentation, we conducted tact training by providing a model prompt, which the child repeated. We then re-presented the trial, giving the child the opportunity to make an independent response. Tact training was complete when child independently tacted the noun or verb three times in a row.

Baseline In baseline, we presented all 162 videos one time and asked, "What?", which had been effective in evoking attempted tacts for these children in the past. The video repeated until the child made a response, but we did not provide reinforcers or feedback during this phase. After every one or two trials, we requested and reinforced a previously mastered response, such as oneword tacts or listener responses.

Matrix Training We selected the first of six matrices at random and trained three $\mathrm{S}-\mathrm{V}-\mathrm{O}$ sentences along a diagonal of that matrix until mastery, reinforcing correct sentence production with access to videos, toys, or 
Table 1 Participant characteristics

\begin{tabular}{|c|c|c|c|c|c|}
\hline Participants & Age & Gender & $\begin{array}{l}\text { VB-MAPP } \\
\text { score }\end{array}$ & Tact repertoire & Mand repertoire \\
\hline Shreeya & $\begin{array}{l}5 \text { years, } \\
2 \text { months }\end{array}$ & Female & 130.5 & $\begin{array}{l}\text { - Tacts at least } 50 \text { items } \\
\text { - Tacts at least } 10 \text { actions } \\
\text { - Tacts at least } 50 \text { noun-verb or } \\
\text { verb-noun relations } \\
\text { - Tacts color, shape, and function } \\
\text { of at least five different objects } \\
\text { - Tacts four prepositions/pronouns }\end{array}$ & $\begin{array}{l}\text { - Mands for at least } 20 \text { different missing } \\
\text { items without prompts } \\
\text { - Mands for others to emit at least five } \\
\text { different actions } \\
\text { - Emits at least five different mands } \\
\text { containing two words or more } \\
\text { - Spontaneously emits at least } 15 \text { different } \\
\text { mands } \\
\text { - Emits at least } 10 \text { different mands without } \\
\text { training } \\
\text { - Spontaneously mands using a WH } \\
\text { question at least two times } \\
\text { - Mands to stop an undesirable activity } \\
\text { under at least two different circumstances }\end{array}$ \\
\hline Jake & $\begin{array}{l}5 \text { years, } \\
7 \text { months }\end{array}$ & Male & 95.5 & $\begin{array}{l}\text { - Tacts at least } 50 \text { items } \\
\text { - Tacts at least } 10 \text { actions } \\
\text { - Tacts at least } 50 \text { noun-verb or } \\
\text { verb-noun relations }\end{array}$ & $\begin{array}{l}\text { - Mands for at least } 20 \text { different missing } \\
\text { items without prompts } \\
\text { - Mands for others to emit at least five } \\
\text { different actions } \\
\text { - Emits at least five different mands } \\
\text { containing two words or more } \\
\text { - Spontaneously emits at least } 15 \text { different } \\
\text { mands }\end{array}$ \\
\hline
\end{tabular}

edibles. Incorrect responses were followed by a model prompt, and the trial was repeated until a correct, independent response occurred. Each session included five three-trial blocks with each of the three training stimuli presented in a random sequence without replacement.

Matrix training was divided into three phases. In the first phase, the experimenter presented a video, said "What?", and immediately provided a model (e.g., "Chase drinks milk"). If the child echoed the model within three seconds, the response was reinforced. In the second phase, we provided a model after a 3-s delay. The child could respond either before or after the model in order for the response to be correct and reinforced. In the third and final phases, the child was required to make an independent response within $3 \mathrm{~s}$ of the "What?" in order for it to be correct and reinforced. If the child did not respond within $3 \mathrm{~s}$, it was incorrect. A model was provided, and the response was then reinforced. Children met mastery criterion in each phase if they scored at or above $93 \%(14 / 15)$ correct in the first session of each phase or if they scored at or above $87 \%$ (13/15) during two consecutive sessions.

Generativity Within Matrices When a child met a mastery criterion for the sentences along a diagonal of a matrix, we tested for generative transfer to the other 24 untrained sentences within that matrix by probing each response one time. A child met criterion for generativity within a matrix if he or she emitted the correct sentence on $92 \%(22 / 24)$ of the trials. This was similar to baseline testing, with no prompts or consequences, along with a reinforced, high-probability instruction every one or two trials. If the child did not meet criterion for generativity within the matrix, we conducted additional training until he or she met the mastery criterion.

Generativity Across Matrices When a child met mastery criterion on all sentences within a matrix, we tested for generative transfer across matrices by testing three sentences along a diagonal of each remaining matrix. If the child responded correctly on fewer than two of the three sentences along the diagonal of one or more of those matrices, we trained along the diagonal of one of these matrices. We repeated the previous steps until the child met criterion for generativity within that matrix and again tested for generativity across the remaining matrices.

If a child responded correctly on at least two of the three sentences along a diagonal, we tested the remaining 24 sentences in that matrix. If the participant met the 


\begin{tabular}{|c|c|c|c|c|c|c|c|c|c|c|c|}
\hline \multicolumn{4}{|c|}{ JESSICA } & \multicolumn{4}{|c|}{ STEVE } & \multicolumn{4}{|c|}{ KHRYSTLE } \\
\hline EATS & $\begin{array}{c}\text { Jessica } \\
\text { eats } \\
\text { banana }\end{array}$ & $\begin{array}{c}\text { Jessica } \\
\text { eats cake }\end{array}$ & EATS & EATS & $\begin{array}{c}\text { Steve eats } \\
\text { banana }\end{array}$ & $\begin{array}{c}\text { Steve eats } \\
\text { cake }\end{array}$ & $\begin{array}{c}\text { Steve eats } \\
\text { apple }\end{array}$ & EATS & $\begin{array}{c}\text { Khrystle } \\
\text { eats } \\
\text { banana }\end{array}$ & $\begin{array}{l}\text { Khrystle } \\
\text { eats cake }\end{array}$ & $\begin{array}{c}\text { Khrystle } \\
\text { eats apple }\end{array}$ \\
\hline SMELLS & $\begin{array}{l}\text { Jessica } \\
\text { smells } \\
\text { banana }\end{array}$ & $\begin{array}{l}\text { Jessica } \\
\text { smells } \\
\text { cake }\end{array}$ & $S M E L L S$ & SMELLS & $\begin{array}{c}\text { Steve } \\
\text { smells } \\
\text { banana }\end{array}$ & $\begin{array}{l}\begin{array}{c}\text { Steve } \\
\text { smells } \\
\text { cake }\end{array} \\
\end{array}$ & $\begin{array}{l}\text { Steve } \\
\text { smells } \\
\text { apple }\end{array}$ & SMELLS & $\begin{array}{c}\text { Khrystle } \\
\text { smells } \\
\text { banana }\end{array}$ & $\begin{array}{c}\text { Khrystle } \\
\text { smells } \\
\text { cake }\end{array}$ & $\begin{array}{c}\text { Khrystle } \\
\text { smells } \\
\text { apple }\end{array}$ \\
\hline \multirow[t]{2}{*}{ CUTS } & $\begin{array}{c}\text { Jessica } \\
\text { cuts } \\
\text { banana }\end{array}$ & $\begin{array}{c}\text { Jessica } \\
\text { cuts cake }\end{array}$ & CUTS & CUTS & $\begin{array}{c}\text { Steve cuts } \\
\text { banana }\end{array}$ & $\begin{array}{c}\text { Steve cuts } \\
\text { cake }\end{array}$ & $\begin{array}{c}\text { Steve cuts } \\
\text { apple }\end{array}$ & CUTS & $\begin{array}{c}\text { Khrystle } \\
\text { cuts } \\
\text { banana }\end{array}$ & $\begin{array}{l}\text { Khrystle } \\
\text { cuts cake }\end{array}$ & $\begin{array}{c}\text { Khrystle } \\
\text { cuts apple }\end{array}$ \\
\hline & BANANA & CAKE & APPLE & & BANANA & CAKE & APPLE & & BANANA & CAKE & APPLE \\
\hline \multicolumn{4}{|c|}{ KELLY } & \multicolumn{4}{|c|}{ LISA } & \multicolumn{4}{|c|}{ JENN } \\
\hline KISSES & $\begin{array}{c}\text { Kelly } \\
\text { kisses cat }\end{array}$ & $\begin{array}{l}\text { Kelly } \\
\text { kisses } \\
\text { Diego }\end{array}$ & $\begin{array}{c}\text { Kelly } \\
\text { kisses } \\
\text { baby }\end{array}$ & KISSES & $\begin{array}{c}\text { Lisa } \\
\text { kisses cat }\end{array}$ & $\begin{array}{c}\text { Lisa } \\
\text { kisses } \\
\text { Diego }\end{array}$ & $\begin{array}{c}\text { Lisa } \\
\text { kisses } \\
\text { baby }\end{array}$ & KISSES & $\begin{array}{c}\text { Jenn } \\
\text { kisses cat }\end{array}$ & $\begin{array}{c}\text { Jenn } \\
\text { kisses } \\
\text { Diego } \\
\end{array}$ & $\begin{array}{c}\text { Jenn } \\
\text { kisses } \\
\text { baby }\end{array}$ \\
\hline$H U G S$ & $\begin{array}{c}\text { Kelly } \\
\text { hugs cat }\end{array}$ & $\begin{array}{l}\text { Kelly } \\
\text { hugs } \\
\text { Diego } \\
\end{array}$ & $\begin{array}{c}\text { Kelly } \\
\text { hugs baby }\end{array}$ & HUGS & $\begin{array}{c}\begin{array}{c}\text { Lisa hugs } \\
\text { cat }\end{array} \\
\end{array}$ & $\begin{array}{c}\text { Lisa hugs } \\
\text { Diego }\end{array}$ & $\begin{array}{c}\text { Lisa hugs } \\
\text { baby }\end{array}$ & $H U G S$ & $\begin{array}{c}\text { Jenn hugs } \\
\text { cat }\end{array}$ & $\begin{array}{c}\text { Jenn hugs } \\
\text { Diego }\end{array}$ & $\begin{array}{c}\text { Jenn hugs } \\
\text { baby }\end{array}$ \\
\hline \multirow[t]{2}{*}{ FEEDS } & $\begin{array}{c}\text { Kelly } \\
\text { feeds cat }\end{array}$ & $\begin{array}{l}\text { Kelly } \\
\text { feeds } \\
\text { Diego }\end{array}$ & $\begin{array}{c}\text { Kelly } \\
\text { feeds baby }\end{array}$ & FEEDS & $\begin{array}{c}\text { Lisa feeds } \\
\text { cat }\end{array}$ & $\begin{array}{c}\text { Lisa feeds } \\
\text { Diego }\end{array}$ & $\begin{array}{c}\text { Lisa feeds } \\
\text { baby }\end{array}$ & FEEDS & $\begin{array}{c}\text { Jenn feeds } \\
\text { cat }\end{array}$ & $\begin{array}{c}\text { Jenn feeds } \\
\text { Diego }\end{array}$ & $\begin{array}{c}\text { Jenn feeds } \\
\text { baby }\end{array}$ \\
\hline & $C A T$ & DIEGO & $B A B Y$ & & $C A T$ & DIEGO & $B A B Y$ & & CAT & DIEGO & $B A B Y$ \\
\hline
\end{tabular}

\begin{tabular}{|c|c|c|c|c|c|c|c|c|c|c|c|}
\hline \multicolumn{4}{|c|}{ WOMAN } & \multicolumn{4}{|c|}{ BABY } & \multicolumn{4}{|c|}{ MAN } \\
\hline SITS & $\begin{array}{c}\text { Woman } \\
\text { sits table }\end{array}$ & $\begin{array}{l}\text { Woman } \\
\text { sits bed }\end{array}$ & $\begin{array}{c}\text { Woman } \\
\text { sits couch }\end{array}$ & SITS & $\begin{array}{c}\text { Baby sits } \\
\text { table }\end{array}$ & $\begin{array}{c}\begin{array}{c}\text { Baby sits } \\
\text { bed }\end{array} \\
\end{array}$ & $\begin{array}{c}\begin{array}{c}\text { Baby sits } \\
\text { couch }\end{array} \\
\end{array}$ & SITS & $\begin{array}{c}\text { Man sits } \\
\text { table }\end{array}$ & $\begin{array}{c}\begin{array}{c}\text { Man sits } \\
\text { bed }\end{array} \\
\end{array}$ & $\begin{array}{c}\text { Man sits } \\
\text { couch }\end{array}$ \\
\hline JUMPS & $\begin{array}{l}\text { Woman } \\
\text { jumps } \\
\text { table }\end{array}$ & $\begin{array}{c}\text { Woman } \\
\text { jumps bed }\end{array}$ & $\begin{array}{l}\text { Woman } \\
\text { jumps } \\
\text { couch } \\
\end{array}$ & JUMPS & $\begin{array}{c}\text { Baby } \\
\text { jumps } \\
\text { table } \\
\end{array}$ & $\begin{array}{c}\text { Baby } \\
\text { jumps bed }\end{array}$ & $\begin{array}{l}\text { Baby } \\
\text { jumps } \\
\text { couch }\end{array}$ & JUMPS & $\begin{array}{c}\text { Man } \\
\text { jumps } \\
\text { table }\end{array}$ & $\begin{array}{c}\text { Man } \\
\text { jumps bed }\end{array}$ & $\begin{array}{c}\text { Man } \\
\text { jumps } \\
\text { couch }\end{array}$ \\
\hline LAYS & $\begin{array}{r}\begin{array}{c}\text { Woman } \\
\text { lays table }\end{array} \\
\end{array}$ & $\begin{array}{l}\text { Woman } \\
\text { lays bed }\end{array}$ & $\begin{array}{c}\text { Woman } \\
\text { lays couch }\end{array}$ & LAYS & $\begin{array}{c}\text { Baby lays } \\
\text { table }\end{array}$ & $\begin{array}{c}\text { Baby lays } \\
\text { bed }\end{array}$ & $\begin{array}{c}\text { Baby lays } \\
\text { couch }\end{array}$ & LAYS & $\begin{array}{c}\text { Man lays } \\
\text { table }\end{array}$ & $\begin{array}{c}\text { Man lays } \\
\text { bed }\end{array}$ & $\begin{array}{c}\text { Man lays } \\
\text { couch }\end{array}$ \\
\hline & TABLE & $B E D$ & $\mathrm{COUCH}$ & & TABLE & $B E D$ & $\mathrm{COUCH}$ & & $T A B L E$ & $B E D$ & $\mathrm{COUCH}$ \\
\hline
\end{tabular}

\begin{tabular}{|c|c|c|c|c|c|c|c|c|c|c|c|}
\hline \multicolumn{4}{|c|}{ CHASE } & \multicolumn{4}{c|}{ MEGAN } & \multicolumn{4}{c|}{ TIM } \\
\hline SPILLS & $\begin{array}{c}\text { Chase } \\
\text { spills milk }\end{array}$ & $\begin{array}{c}\text { Chase } \\
\text { spills juice }\end{array}$ & $\begin{array}{c}\text { Chase } \\
\text { spills } \\
\text { water }\end{array}$ & SPILLS & $\begin{array}{c}\text { Megan } \\
\text { spills milk }\end{array}$ & $\begin{array}{c}\text { Megan } \\
\text { spills juice }\end{array}$ & $\begin{array}{c}\text { Megan } \\
\text { spills } \\
\text { water }\end{array}$ & SPILLS & $\begin{array}{c}\text { Tim spills } \\
\text { milk }\end{array}$ & $\begin{array}{c}\text { Tim spills } \\
\text { juice }\end{array}$ & $\begin{array}{c}\text { Tim spills } \\
\text { water }\end{array}$ \\
\hline DRINKS & $\begin{array}{c}\text { Chase } \\
\text { drinks } \\
\text { milk }\end{array}$ & $\begin{array}{c}\text { Chase } \\
\text { drinks } \\
\text { juice }\end{array}$ & $\begin{array}{c}\text { Chase } \\
\text { drinks } \\
\text { water }\end{array}$ & DRINKS & $\begin{array}{c}\text { Megan } \\
\text { drinks } \\
\text { milk }\end{array}$ & $\begin{array}{c}\text { Megan } \\
\text { drinks } \\
\text { juice }\end{array}$ & $\begin{array}{c}\text { Megan } \\
\text { drinks } \\
\text { water }\end{array}$ & DRINKS & $\begin{array}{c}\text { Tim } \\
\text { drinks } \\
\text { milk }\end{array}$ & $\begin{array}{c}\text { Tim } \\
\text { drinks } \\
\text { juice }\end{array}$ & $\begin{array}{c}\text { Tim } \\
\text { drinks } \\
\text { water }\end{array}$ \\
\hline STIRS & $\begin{array}{c}\text { Chase } \\
\text { stirs milk }\end{array}$ & $\begin{array}{c}\text { Chase } \\
\text { stirs juice }\end{array}$ & $\begin{array}{c}\text { Chase } \\
\text { stirs water }\end{array}$ & STIRS & $\begin{array}{c}\text { Megan } \\
\text { stirs milk }\end{array}$ & $\begin{array}{c}\text { Megan } \\
\text { stirs juice }\end{array}$ & $\begin{array}{c}\text { Megan } \\
\text { stirs juice }\end{array}$ & STIRS & $\begin{array}{c}\text { Tim stirs } \\
\text { milk }\end{array}$ & $\begin{array}{c}\text { Timm stirs } \\
\text { juice }\end{array}$ & $\begin{array}{c}\text { Tim stirs } \\
\text { water }\end{array}$ \\
\hline & MILK & JUICE & WATER & & MILK & JUICE & WATER & & MILK & JUICE & WATER \\
\hline
\end{tabular}

\begin{tabular}{|c|c|c|c|c|c|c|c|c|c|c|c|}
\hline \multicolumn{4}{|c|}{ SHREEYA } & \multicolumn{4}{|c|}{ EMILIA } & \multicolumn{4}{|c|}{ MORGAN } \\
\hline OPENS & $\begin{array}{l}\text { Shreeya } \\
\text { opens } \\
\text { locker }\end{array}$ & $\begin{array}{c}\begin{array}{c}\text { Shreeya } \\
\text { opens } \\
\text { microwave }\end{array} \\
\end{array}$ & $\begin{array}{c}\text { Shreeya } \\
\text { opens } \\
\text { door }\end{array}$ & OPENS & $\begin{array}{l}\text { Emilia } \\
\text { opens } \\
\text { locker }\end{array}$ & $\begin{array}{c}\begin{array}{c}\text { Emilia } \\
\text { opens } \\
\text { microwave }\end{array} \\
\end{array}$ & $\begin{array}{c}\text { Emilia } \\
\text { opens } \\
\text { door }\end{array}$ & OPENS & $\begin{array}{c}\text { Morgan } \\
\text { opens } \\
\text { locker } \\
\end{array}$ & $\begin{array}{c}\text { Morgan } \\
\text { opens } \\
\text { microwave }\end{array}$ & $\begin{array}{c}\text { Morgan } \\
\text { opens } \\
\text { door }\end{array}$ \\
\hline CLOSES & $\begin{array}{l}\text { Shreeya } \\
\text { closes } \\
\text { locker }\end{array}$ & $\begin{array}{c}\begin{array}{c}\text { Shreeya } \\
\text { closes } \\
\text { microwave }\end{array} \\
\text { misolo }\end{array}$ & $\begin{array}{l}\text { Shreeya } \\
\text { closes } \\
\text { door }\end{array}$ & CLOSES & $\begin{array}{l}\text { Emilia } \\
\text { closes } \\
\text { locker }\end{array}$ & $\begin{array}{c}\text { Emilia } \\
\text { closes } \\
\text { microwave }\end{array}$ & $\begin{array}{l}\text { Emilia } \\
\text { closes } \\
\text { door }\end{array}$ & CLOSES & $\begin{array}{c}\text { Morgan } \\
\text { closes } \\
\text { locker }\end{array}$ & $\begin{array}{c}\text { Morgan } \\
\text { closes } \\
\text { microwave }\end{array}$ & $\begin{array}{c}\text { Morgan } \\
\text { closes } \\
\text { door }\end{array}$ \\
\hline KNOCKS & $\begin{array}{l}\text { Shreeya } \\
\text { knocks } \\
\text { locker } \\
\end{array}$ & $\begin{array}{c}\text { Shreeya } \\
\text { knocks } \\
\text { microwave }\end{array}$ & $\begin{array}{c}\text { Shreeya } \\
\text { knocks } \\
\text { door }\end{array}$ & KNOCKS & $\begin{array}{l}\text { Emilia } \\
\text { knocks } \\
\text { locker } \\
\end{array}$ & $\begin{array}{c}\text { Emilia } \\
\text { knocks } \\
\text { microwave }\end{array}$ & $\begin{array}{c}\begin{array}{c}\text { Emilia } \\
\text { knocks } \\
\text { door }\end{array} \\
\end{array}$ & KNOCKS & $\begin{array}{l}\text { Morgan } \\
\text { knocks } \\
\text { locker } \\
\end{array}$ & $\begin{array}{c}\text { Morgan } \\
\text { knocks } \\
\text { microwave }\end{array}$ & $\begin{array}{c}\text { Morgan } \\
\text { knocks } \\
\text { door } \\
\end{array}$ \\
\hline & LOCKER & MICROWAVE & DOOR & & LOCKER & MICROWAVE & DOOR & & LOCKER & MICROWAVE & DOOR \\
\hline
\end{tabular}

\begin{tabular}{|c|c|c|c|c|c|c|c|c|c|c|c|}
\hline \multicolumn{4}{|c|}{ HUNTER } & \multicolumn{4}{|c|}{ JAKE } & \multicolumn{4}{|c|}{ CONOR } \\
\hline KICKS & $\begin{array}{c}\begin{array}{c}\text { Hunter } \\
\text { kicks ball }\end{array} \\
\end{array}$ & $\begin{array}{l}\text { Hunter } \\
\text { kicks } \\
\text { block } \\
\end{array}$ & $\begin{array}{c}\text { Hunter } \\
\text { kicks } \\
\text { book } \\
\end{array}$ & KICKS & $\begin{array}{c}\text { Jake kicks } \\
\text { ball }\end{array}$ & $\begin{array}{c}\text { Jake kicks } \\
\text { block }\end{array}$ & $\begin{array}{c}\text { Jake kicks } \\
\text { book }\end{array}$ & KICKS & $\begin{array}{c}\text { Conor } \\
\text { kicks ball }\end{array}$ & $\begin{array}{l}\text { Conor } \\
\text { kicks } \\
\text { block } \\
\end{array}$ & 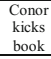 \\
\hline THROWS & $\begin{array}{c}\text { Hunter } \\
\text { throws } \\
\text { ball }\end{array}$ & $\begin{array}{l}\text { Hunter } \\
\text { throws } \\
\text { block }\end{array}$ & $\begin{array}{c}\text { Hunter } \\
\text { throws } \\
\text { book }\end{array}$ & THROWS & $\begin{array}{c}\text { Jake } \\
\text { throws } \\
\text { ball } \\
\end{array}$ & $\begin{array}{c}\text { Jake } \\
\text { throws } \\
\text { block } \\
\end{array}$ & $\begin{array}{c}\text { Jake } \\
\text { throws } \\
\text { book } \\
\end{array}$ & THROWS & $\begin{array}{c}\text { Conor } \\
\text { throws } \\
\text { ball } \\
\end{array}$ & $\begin{array}{l}\text { Conor } \\
\text { throws } \\
\text { block } \\
\end{array}$ & $\begin{array}{c}\text { Conor } \\
\text { throws } \\
\text { book } \\
\end{array}$ \\
\hline DROPS & $\begin{array}{c}\text { Hunter } \\
\text { drops ball }\end{array}$ & $\begin{array}{c}\text { Hunter } \\
\text { drops } \\
\text { block } \\
\end{array}$ & $\begin{array}{c}\begin{array}{c}\text { Hunter } \\
\text { drops } \\
\text { book }\end{array} \\
\end{array}$ & DROPS & $\begin{array}{c}\begin{array}{c}\text { Jake drops } \\
\text { ball }\end{array} \\
\end{array}$ & $\begin{array}{c}\begin{array}{c}\text { Jake drops } \\
\text { block }\end{array} \\
\end{array}$ & $\begin{array}{c}\text { Jake drops } \\
\text { book }\end{array}$ & DROPS & $\begin{array}{c}\text { Conor } \\
\text { drops ball }\end{array}$ & $\begin{array}{l}\begin{array}{l}\text { Conor } \\
\text { drops } \\
\text { block }\end{array} \\
\end{array}$ & $\begin{array}{l}\begin{array}{l}\text { Conor } \\
\text { drops } \\
\text { book }\end{array} \\
\end{array}$ \\
\hline & $B A L L$ & $B L O C K$ & $B O O K$ & & $B A L L$ & $\overline{B L O C K}$ & BOOK & & $B A L L$ & $B L O C K$ & BOOK \\
\hline
\end{tabular}

Fig. 1 Subjects are listed above the matrices; verbs are listed vertically and objects horizontally. Shaded cells indicate responses along the "diagonal" of a matrix, which allows for each word to be used one time without overlap

generativity criterion, he or she had demonstrated generative transfer across matrices. If not, we conducted additional training sessions with that matrix until the child met the mastery criterion.

During some of the probes, we observed that Jake's incorrect responses might be due to poor attending. When this occurred, we re-probed the trials that were incorrect, and the new, combined score is indicated by a dotted square on the graph.

Additional Training When behavior did not meet criterion for generativity within or across matrices, we conducted additional training. The additional training format was identical to the final phase of matrix training.
Because Shreeya's errors involved incorrect verbs but correct sentence structure, we only conducted additional training with the sentences that had occasioned incorrect responses during the generativity probes. However, Jake's errors were less consistent, so we conducted additional training on all of the non-diagonal responses within a particular matrix. Each target was presented in random order, once per training session. A child met the mastery criterion during the additional training sessions when he or she made no more than two errors on each of two consecutive sessions.

Maintenance Probes We conducted maintenance probes 8 months after training for Shreeya and 
3 months after training for Jake. To the best of our knowledge, no training with these sentences had occurred during the intervening months. The probes were similar to the previous probes, except that we provided prompts following incorrect responses. The incorrect trials were repeated along with the prompts (as needed) until the child made a correct, independent response. Other than the prompts, we did not provide any additional feedback for correct or incorrect responses.

\section{Results and Discussion}

Neither child made any correct responses during baseline, although both frequently tacted a single component of the video. After three sessions of training, Shreeya began demonstrating a generative $\mathrm{S}-\mathrm{V}-\mathrm{O}$ repertoire (Fig. 2). In matrix 4 and matrix 6, she required additional training, but not on the sentence structure. The errors she made on these probes were incorrect tacts of the actions ("drinks" instead of "feeds" and "drops" instead of "spills"). In total, Shreeya mastered all 162 sentences within 24 sessions and received explicit training on only 14 sentences. Thus, she demonstrated generative transfer across the five remaining matrices, except the two problem verbs.

Jake required more training than Shreeya before demonstrating a generative S-V-O repertoire (Fig. 3). In total, Jake mastered all 162 sentences within 37 sessions, with explicit training on 78 of those sentences. He required 32 sessions of explicit training across four

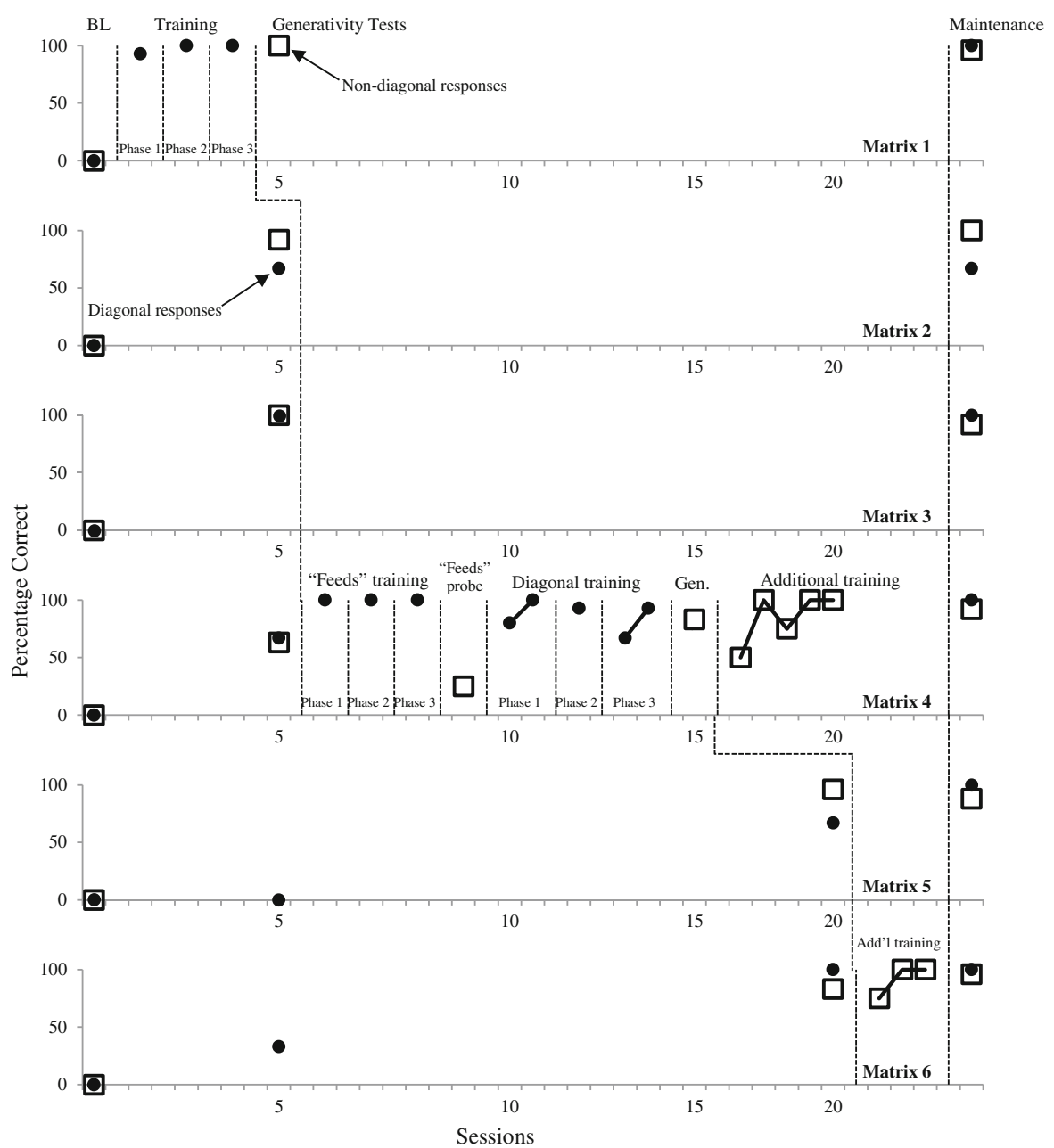

Fig. 2 The results of matrix training for Shreeya. Closed circles represent responses along the diagonal of each matrix, and open squares represent non-diagonal responses. BL refers to baseline, and Gen. refers to the tests for generativity 

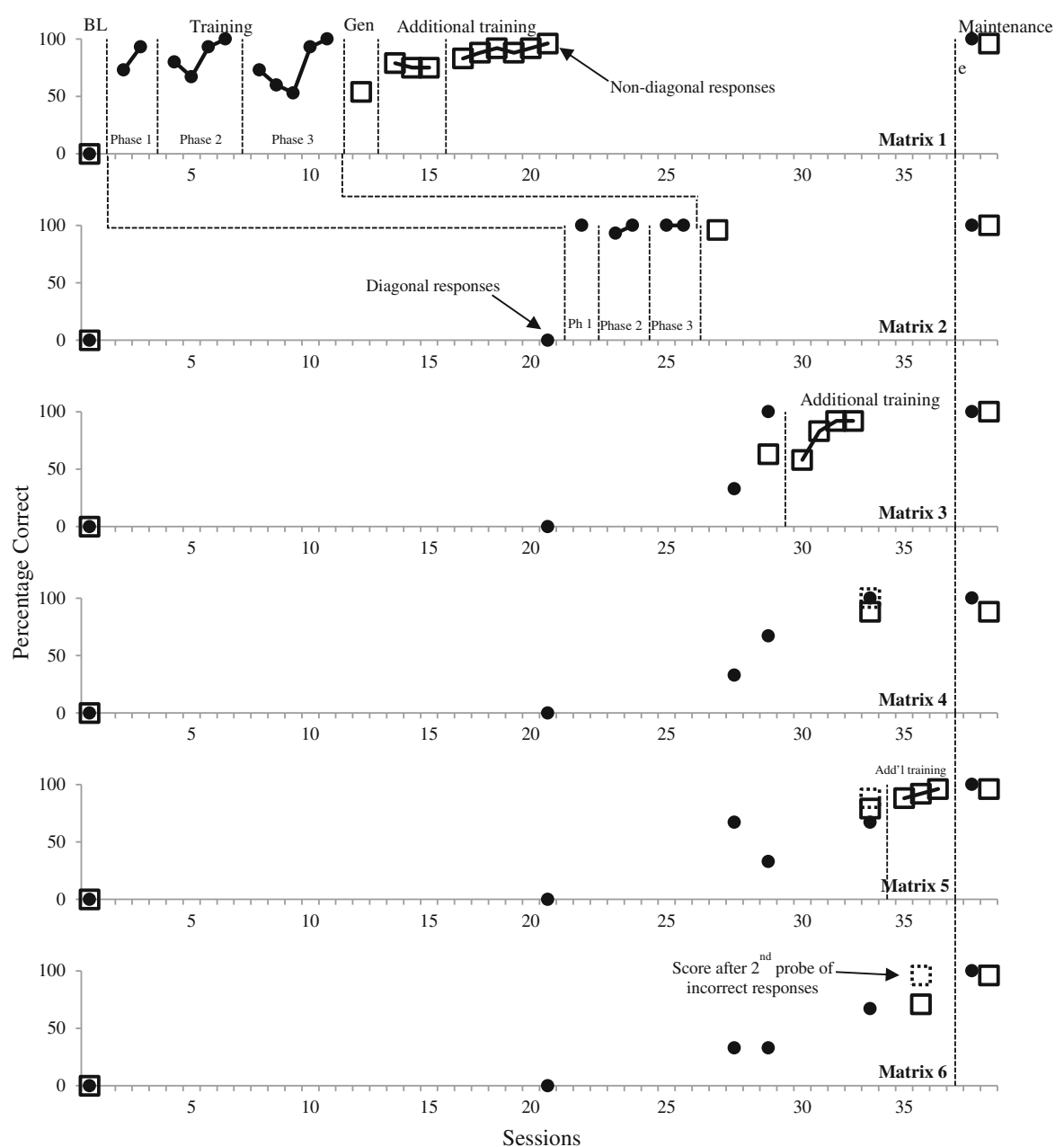

Fig. 3 The results of matrix training for Jake. Closed circles represent responses along the diagonal of each matrix, and open squares represent non-diagonal responses. Dotted squares

matrices for generative transfer to the remaining two matrices.

During the maintenance probes, Shreeya responded correctly on 24 to 26 out of 27 trials, and Jake responded correctly on 24 to 27 out of 27 trials on each of the matrices, demonstrating that the skill had maintained for represent the non-diagonal score after a second probe of the incorrect responses. $B L$ refers to baseline, and Gen. refers to the tests for generativity

both children. Though not tested formally, both children demonstrated transfer to novel S-V-O sentences that were not a part of the training or testing procedure. In a less structured play setting, we informally assessed whether they could receptively follow instructions given in an S-V-O format (e.g., "show me cow eats carrot")

Table 2 Summary of results

\begin{tabular}{llllll}
\hline Participants & $\begin{array}{l}\text { Number of individual tacts needing } \\
\text { training out of } 53^{\mathrm{a}} \text { used }\end{array}$ & $\begin{array}{l}\text { S-V-O tacts directly } \\
\text { trained }\end{array}$ & $\begin{array}{l}\text { Untrained S-V-O } \\
\text { tacts acquired }\end{array}$ & $\begin{array}{l}\text { Sessions to } \\
\text { mastery }\end{array}$ & $\begin{array}{l}\text { Number of correct responses } \\
\text { during maintenance probe }\end{array}$ \\
\hline Shreeya & 11 & $14 / 162$ & 148 & 24 & $152 / 162$ \\
Jake & 28 & $78 / 162$ & 84 & 37 & $156 / 162$ \\
\hline
\end{tabular}

a The word "baby" was used twice — once as a subject and once as an object 
and whether they could tact untrained S-V-O combinations (e.g., "pig kisses cow"). They were successful with both.

The current study evaluated the effects of matrix training on the acquisition of generative subject-verb-object (S-V-O) sentences. At the start of this study, the two children could label the individual components of all of the S-V-O sentences that were to be used, but they did not correctly form S-V-O sentences. Matrix training targets each component within a matrix without overlap, which results in very efficient teaching. Often, a child is then able to use the trained sentence structure when tacting untrained combinations within and across matrices. After training with 14 (Shreeya) and 78 (Jake) sentences, the two children in this study demonstrated transfer to the remaining 148 or 84 sentences (see Table 2), suggesting that they had acquired a generalized S-V-O sentence structure. This is consistent with previous findings demonstrating that training on subset of responses can result in the acquisition of several related, untrained responses (e.g., Axe and Sainato 2010; Goldstein 1983a; Goldstein 1983b; Goldstein 1984; Goldstein and Brown 1989; Goldstein and Mousetis 1989; Yamamoto and Miya 1999).

An informal analysis of prerequisite skills and reinforcers might explain the differences between the two participants in acquisition of the generative S-V-O sentences. Computers, videos, and other forms of media seemed to be powerful reinforcers for Shreeya, so the procedure itself was probably more reinforcing. These stimuli seemed to be less reinforcing for Jake. Also, Jake engaged in a high rate of interfering stereotypy during training and testing.

Both children entered the study with similar tact repertoires. However, the relevant tacts in the children's repertoire at the beginning of this study may also have contributed to their differences in speed of acquisition. Shreeya only needed additional tact training on 11 of the 53 words to be used in the study, while Jake needed training on 28 tacts (see Table 2).

Also, we noted that neither child spontaneously used these S-V-O tacts outside the experimental sessions, perhaps because tacts are normally maintained by social reinforcers, such as praise or attention (Skinner 1957, p. 83), and for these two children, social events may not have been effective reinforcers. Therefore, our laboratory is currently doing research on establishing social reinforcers that can maintain tacting, including S-V-O tacts, during naturalistic interactions.
The primary limitation of the current study is the lack of consistent demonstration of experimental control through the multiple probe design because both participants demonstrated acquisition across matrixes following training with one or two matrixes. Thus, control for common threats to internal validity (e.g., history and testing) is limited.

We used an approximation of a non-concurrent multiple baseline design across participants, as we did not bring Jake into the study until Shreeya had finished. However, we did not vary the number of baseline sessions across participants. Future studies could rectify this limitation by incorporating repeated baseline probes prior to teaching the first matrix and varying the number of such probes across participants. Further, future research could also use a concurrent multiple baseline design across participants.

\section{References}

Axe, J. B., \& Sainato, D. M. (2010). Matrix training of preliteracy skills with preschoolers with autism. Journal of Applied Behavior Analysis, 43(4), 635-652.

Chomsky, N. (1959). A review of B.F. Skinner's Verbal Behavior. Language, 35, 26-58.

Dauphin, M., Kinney, E. M., \& Stromer, R. (2004). Using videoenhanced activity schedules and matrix training to teach sociodramatic play to a child with autism. Journal of Positive Behavior Interventions, 6(4), 238-250.

Esper, E. A. (1925). A technique for the experimental investigation of associative interference in artificial linguistic material. Language Monographs, 1, 5-46.

Foss, D. J. (1968). Learning and discovery in the acquisition of structured material: effects of number of items and their sequence. Journal of Experimental Psychology, 77(2), 341344.

Goldstein, H. (1983a). Recombinative generalization: relationships between environmental conditions and the linguistic repertoires of language learners. Analysis and Intervention in Developmental Disabilities, 3, 279-293.

Goldstein, H. (1983b). Training generative repertoires within agent-action-object miniature linguistic systems with children. Journal of Speech and Hearing Research, 26, 76-89.

Goldstein, H. (1984). Effects of modeling and corrected practice on generative language learning of preschool children. Journal of Speech and Hearing Research, 49, 389-398.

Goldstein, H., \& Brown, W. H. (1989). Observational learning of receptive and expressive language by handicapped preschool children. Education and Treatment of Children, 12, 5-37.

Goldstein, H., \& Mousetis, L. (1989). Generalized language learning by children with severe mental retardation: effects of peers' expressive modeling. Journal of Applied Behavior Analysis, 22, 245-259. 
Hockett, C. F. (1960). The origin of speech. Scientific American, 203, 88-96.

Lutzker, J. R., \& Sherman, J. A. (1974). Producing generative sentence usage by imitation and reinforcement procedures. Journal of Applied Behavior Analysis, 7(3), 447-460.

Mackay, H. A., \& Fields, L. (2009). Syntax, grammatical transformation, and productivity: a synthesis of stimulus sequences, equivalence classes and contextual control. In R. A. Rehfeldt \& Y. Barnes-Holmes (Eds.), Derived relational responding: applications for learners with autism and other developmental disabilities: A progressive guide for change (pp. 209-236). London: New Harbinger.

Malott, R. W. (2003). Behavior analysis and linguistic productivity. The Analysis of Verbal Behavior, 19, 11-18.
Malott, R., Fronapfel-Sonderegger, B., Perry, K., Shane, J., Stone, K., \& Korneder. (2011, January). A practitioner model for undergraduate and graduate training in autism. Paper presented at the Association for Behavior Analysis Fifth Annual Autism Conference, Washington, DC.

Skinner, B. F. (1957). Verbal behavior. New York: AppletonCentury-Croft.

Stewart, I., McElwee, J., \& Ming, S. (2013). Language generativity, response generalization, and derived relational responding. The Analysis of Verbal Behavior, 29, 137-155.

Whaley, D. L., \& Malott, R. W. (1971). Elementary principles of behavior (1st ed.). Upper Saddle River: Prentice Hall.

Yamamoto, J., \& Miya, T. (1999). Acquisition and transfer of sentence construction in autistic students: analysis by computer-based teaching. Research in Developmental Disabilities, 20(5), 355-377. 